\title{
Structural and dynamical analysis of complex networks
}

\author{
Djibril Mboup ${ }^{1,2, a, *}$, Cherif Diallo ${ }^{1,3, b}$, and Moussa Lo $\mathrm{o}^{1,2, \mathrm{c}}$ \\ ${ }^{1}$ Computer Science Department, Gaston Berger University, Senegal \\ ${ }^{2}$ Laboratoire Analyse Numérique et Informatique (LANI) \\ ${ }^{3}$ Laboratoire d'Algèbre Code Crytographie et Application (ACCA)

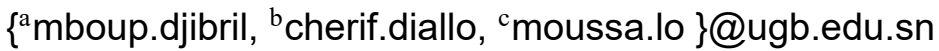

Keywords: complex networks, dynamical networks, evolving networks.

\begin{abstract}
We encounter in real world large networks as diverse as neural networks, power grid, financial networks, friendship networks, Internet, WWW. These networks are commonly characterized by a large number of highly interconnected dynamical entities. They are so complex that they may give birth to many dynamic phenomena. Understanding at the same time the topological and dynamical aspect of complex networks is an important challenge. In this paper, we review on the fundamental elements of complex networks study. We include definitions, measurements, models used to analyse topology and dynamics of these systems. Moreover, we discuss some questions related to mobility and dynamic community detection in complex systems.
\end{abstract}

\section{प[Introduction}

For a long time, networks have been studied with great importance in many domains that are scientific or not. The study of networks has mostly developed in research fields such as social sciences, physics, computer sciences and molecular biology. A network is an abstract representation of entities or elements as regards a domain with nodes (or points) and their relationships (or interactions) with links called edges. Known in the mathematical field as graph, a network can represent for instance the rumour spreading in a social network such as the nodes whose individuals and links represent their diverse relationships, or Internet whose nodes are devices (computers, routers ...) and links are the cables or the air.

The late 1990s witnessed a boom of research activities around the study of networks said 'complex' (i.e. networks whose structure is irregular, complex and dynamically evolving in time [12]) which are, generally, the result of decentralized and planed evolution. Indeed, the study of complex networks has known dramatic advances sustained by the recent appearance of large databases that allow studying systematically the topology of various real networks and the increased computing power allows us to explore networks containing millions of nodes.

Some deficiencies observed in the complex networks modelling based on graph theory has led researchers to introduce new definitions and metrics, which help us to mimic their structure and their dynamic evolution. In spite of many models proposed, the problem concerning the dynamic evolution in a network is a subject which interests researchers in complex networks area. For this reason, we study, analyse and try to understand the two aspects that are the structure and the dynamic evolution of complex networks.

This report is divided into four sections organized as follows:

First, in section 1, we give a description of a complex network in order to explain what is the origin of this science and where do we find it. In section 2, we describe in detail the structure of complex networks by reviewing the different definitions, notations and metrics, and the different benchmark models of networks such as random graphs, small world and the scale free. After, in section 3 , we explain how we understand dynamics in a complex network and we propose some approaches used to model this dynamics. Finally, we exhibit in section 4 some problems and perspectives related such as mobility and community detection in dynamic networks. 


\section{Complex Networks}

\section{प.1. Historical background and definition}

The study of complex networks have attracted great attention within the scientific community during the last decades. The complex network is a part of the great family of complex systems. The notion of complex systems has interested a large body of researchers such as biologists, physicists, sociologists, geographers, computer scientists, to name but a few. Everyone studies these systems in different manners with different tools. Newman in [44] explains that there is no precise technical definition of a 'complex system' but most researchers in the field would probably agree that it is a system composed of many interacting parts called 'agents', such that the collective behaviour of those parts together is more than the sum of their individual behaviours. The collective behaviours are sometimes also called 'emergent' behaviours, and a complex system can thus be said to be a system of interacting parts that displays emergent behaviour. To quantify such a system, at first, you must specify its topology-who interacts with whom-and then its dynamic-how the individual agents behave and how they interact [44].

The theoretical modelling of complex systems can be divided according to Newman between two approaches [44]. The first involves the creation and study of simplified mathematical models that, while they may not mimic the behaviour of real systems exactly, try to abstract the most important qualitative elements into a solvable framework from which we can gain scientific insight. The second approach is to create more comprehensive and realistic models, usually in the form of computer simulations. In this optic, the network become an unavoidable element to model a complex system.

In the recent years, the research in complex networks has known an important event with the works by Watts and Strogatz on small-world networks [63], in 1998, by Barabási and Albert on scale-free networks [6], in 1999. Today, these activities constitute a fundamental element for studying and understanding the properties of a complex network. After these pioneers, a lot of books and articles have appeared in the literature with themes concerning the structure, as well as the dynamics of networks.

It is important to know what a complex network is. Many definitions of complex networks exist in the literature; we try to give some relevant definitions to help us to understand about this system. Newman defines a complex network to be an interacting network between entities, such that the global behaviour of those entities is not deductible to their individual behaviours, we call this phenomenon an emergent behaviour [43]. Moreover, in a presentation titled Applications of Complex Networks [13], Buldú defines a complex network as a network with non-trivial topological features, with patterns of connection between their elements that are neither purely regular nor purely random. According to Boccaletti et al., a complex network is a network whose structure is irregular, complex and dynamically evolving in time [12]. All definitions point out the complexity of its topology, we remark also it is not static but a dynamic system. We can see this dynamic by two sides : the network evolves then nodes appear and/or disappear in time and the dynamic processes take place inside it such as spreading of rumour or disease, diffusion, routing and so on. Examples of complex networks include social networks, power grid, economy networks, chemistry components, neural networks, transportation networks, epidemic spreading, metabolic pathways, mobile networks, food web, Internet, World Wide Web etc.

\section{प्2. Real Networks}

The real networks are the networks of the real world. Researchers of diverse disciplines have explored these networks recently. In [43], Newman suggests to subdivide networks by four categories, which are social networks, information networks, technological networks, and biological networks.

1.2.1. Social Networks. A social network can be defined formally as a set of individuals or social entities linked through some kind of interactions among them [12]. Their interactions could be as diverse as friendships, collaborations, sexual contacts or business relationships. The social network analysis started to develop in the early 1920s with the works of Simmel, Wellman, Bouglé and others. 
Furthermore, it has known a very fast development due to Milgram experience, an American psychologist, who introduced the 'six degrees of separation' in 1967. A classical example of social networks is the collaboration network of film actors such that datasets are available in IMBD (Online Movie Database). In this network, two actors are considered connected if they have played together in the same film. The statistical properties of collaboration network of film actors have been analysed by certain authors [4] [1] [2]. Others examples of social networks have also been studied in scientific community such as co-authors networks in academia, phone call networks, email networks, sexual contact networks and so on.

1.2.2. Information Networks. According to Newman, the information networks have related to the classical example of citation networks between scientific papers [43]. Intuitively, we remark that many articles cite precedent articles on related subjects. In fact, citations between scientific papers constitute a network whose nodes are papers and it exists a direct edge from node $\mathrm{A}$ to node $\mathrm{B}$ indicating that paper A cites paper B. This network is acyclic because one cannot cite an article that does not exist previously [43]. Redner, studying the distribution of 783, 339 citation papers catalogued by the Institute of Scientific Information and 24, 296 papers published in Physical Review D between 1975 and 1994, has found that the probability one paper may be cited $k$ times follows the power-law distribution $\left(p_{k} \sim k^{-\lambda}\right)$ [55]. The World Wide Web (WWW) with their webpages and hyperlinks constitute the most interesting information networks studied in the community. The study of the WWW network have started since the late 1990s with Albert, Jeong, Barabási [5] and Kumar et al. [33].

1.2.3. Technological Networks. Generally, these networks are destined for transportation to service or energy. The first network studied in this category is the topology of electrical power grid network of Western States Power Grid in USA [63]. In this category, others networks have been studied including air transportation, road traffic, railway transportation. The technological network that has mostly attracted our attention is the Internet. The Internet is a network composed of computers and others telecommunication devices, which are connected by cables or waves. The topology of the Internet has been examined in two levels. At the router level, nodes represent routers and links are the physical connections between routers. At the inter-domain (or autonomous) level a single node represents each domain, composed of hundreds of routers and computers, and an edge is drawn between two domains if there is at least one route that connects them. Faloutsos et al. have studied the Internet at both levels, concluding that in each case the degree distribution follows a power-law [22].

1.2.4. Biological Networks. Finally, the last category is the biological networks, which concern all networks related to living beings. The classical example of biological networks is the metabolic pathways. In this network, the substrates (ATP, ADP, H2O ...) are considered as nodes and there are links whether it exist a chemical reaction whose result acts on the substrates. In addition, there are many important classes of biological networks that have interested the community of complex networks, for example protein-protein interaction, genetic regulatory networks, neural networks, food web etc.

\section{Structure of complex networks}

A complex network has a particular structure due to its large dimension (sometimes almost millions of nodes) and its many interactions, which play an important role in its characteristic. Thus, we introduce in this section certain definitions, metrics and notations that allow us to better understand the topology of complex networks. After doing a review of the benchmark models proposed, we show how their properties are approximately different from real-world networks.

\subsection{Definitions, metrics, notations}

Complex networks study is essentially based on the concepts of graph theory. These concepts are not sufficient to do an exhaustive analysis of such system. That is the reason why researchers invent new tools to complete the study based on graph theory. Here, we define the different concepts used in 
complex networks study.

3.1.1 Graph. Mathematically, a graph $G$ is a couple $(V, E)$ where:

- $V$ is a finite set of object. The elements of $V$ are said nodes or vertices.

- $E$ is a subset of $V \times V$. The elements of $E$ are called edges or links.

A graph can be oriented (called directed graph) or not (undirected graph). Sometimes, it may be important to quantify the links by giving a value, which represents the strength or the weight then the graph is said weighted graph. A subgraph $G^{\prime}=\left(V^{\prime}, E^{\prime}\right)$ is a graph such that $V^{\prime} \subseteq V, E^{\prime} \subseteq E$. We encounter many types of graphs according their structure. A multigraph is a graph such that there are multiples edges between two nodes. A bipartite graph is defined as a graph whose nodes can be divided into two separate sets or classes such that every edge links a node of one set to a node of the other set. A graph is simple whether it is not a multigraph and it does not contain loops, i.e. links from a node to itself. Basically, a graph $G=(E, V)$ is exactly equivalent with its adjacent matrix. Let consider $A=\left(a_{i j}\right)$, the adjacent matrix of $G$ such that:

$$
a_{i j}=\left\{\begin{array}{cc}
1 & \text { si } \\
0 & \text { sinon }
\end{array}(i, j) \in E\right.
$$

3.1.2. Degree. In the graph $G$, the vertex $i$ is adjacent (or connected) to another vertex $j$ if it exists a link between $i$ and $j$. It is also common to call connected vertices neighbours. The degree of nodes is the amount of adjacent links. We denote node degree $k_{i}$ given by the following expression:

$$
k_{i}=\sum_{j \in V} a_{i j} ; \text { avec } a_{i j} \in A
$$

In case of directed graphs, we use the notions of in-degree $k^{i n}$ or out-degree $k^{\text {out }}$ of a vertex $i$. The in-degree $k^{i n}$ of a vertex is defined as the sum of arcs, which have the vertex $i$ as final extremity whereas the out-degree $k^{\text {out }}$ is the sum of arcs which quit the node $i$. Consequently, the degree $k_{i}$ of node $i$ may be donated by $k_{i}=k^{i n}+k^{\text {out }}$

3.1.3. Connectivity. Joining a node from any other node constitutes a fundamental aspect in real networks. In a graph $G$, a walk is defined to be a finite and alternative sequence of vertices and edges, beginning and finishing by nodes such that each edge is incident with vertices around it. A cycle is a closed walk. A walk is elementary if all these nodes are distinct whereas a walk is simple if all these edges are also distinct. A path is a walk whose arcs are oriented as directed graphs. A circuit is only a closed path.

A graph $G=(V, E)$ is connected if it exists a path between every pair $(i, j)$ of nodes, otherwise it is disconnected. A connected component stands for a subset of vertices that are connected together. In other words, a connected graph is a graph that have shaped only maximal connected components. Moreover, a giant component is a component whose size is of the same order as $N$. The complete graphs, i.e. graphs such that all-possible pairs of vertices are joined by edges, present always a connected structure. Hence, a complete graph with $\mathrm{N}$ nodes has exactly $K=N(N-1) / 2$ links. In this case, $G$ is noted $K_{N}$. A clique is a complete subgraph.

The Density $D$ of a graph can be defined as the coefficient of the number of existing edges on the all-possible edges in the graph. A graph $G$ is said to be dense if $K=O\left(N^{2}\right)$, contrariwise it is sparse in the case of $D \ll 1$ or $K \ll N^{2}$. We cannot ignore the role of density in the graph connectivity. Naturally, most real networks studied in the literature present a sparse structure.

3.1.4. Shortest path and Average path length. The shortest path or geodesic distance is a fundamental concept in graph theory. Thus, it plays an important role in transportation and communication networks for instance routing protocols in networks such as the Internet [12]. Formally, the shortest path $d_{i j}$ from $i$ to $j$ is the minimum distance of all paths that connect $i$ and $j$. Therefore, the diameter is defined to be the max geodesic path within the graph, $\operatorname{Diam}(G)=\max \left(d_{i j}\right)$. 
The average path length is defined to be the mean of shortest distance between all pairs of nodes in the graph. It is also named mean geodesic distance. Let consider $l$ to be the average path length, mathematically we obtain the measure of $l$ by the following formula:

$$
l=\frac{1}{N(N-1)} \sum_{\substack{i, j \\ i \neq j}} d_{i j}
$$

where $d_{i j}$ is the geodesic distance between $i$ and $j$ and $N$ represents the number of nodes. Unfortunately, this definition is applicable whether the graph is connected i.e. if it exists a path for each pair of nodes. However, if the graph disconnects the measure of $l$ diverge. For this reason, to avoid this problem, it is proposed to use the 'harmonic mean' or global efficiency, denoted $E_{\text {glob }}$ [36].

$$
E_{\text {glob }}(G)=\frac{1}{N(N-1)} \sum_{\substack{i, j \\ i \neq j}} \frac{1}{d_{i j}}
$$

Thus, each pair of vertices taken in a disconnected component participate in the quantity of $E_{\text {glob }}$ with about a null value. We have by definition the inequality $0 \leq E_{\text {glob }}(G) \leq 1$.

3.1.5. Small world effect. We witnessed for the first time, the birth of small world effects after the experience did by the social psychologist, Stanley Milgram. Towards 1960s, Milgram took the Frigyes Karinthy's idea developed in 'Chains' which was written in 1929. The theory said that with any two people chosen randomly in the world, we have six acquaintances average to connect these two people. To test this idea, Milgram gave 300 letters to participants living in Omaha then asked them to send the letters to a change agent living in Boston. The participants must deliver the letter to the target person directly (hand by hand) or by acquaintances. After the experience, Milgram found that the average path in a chain of acquaintances such that one person joins another was six. Thus, this experience is called 'six degree of separation' few years later by John Gaure. This discovery has created a revolution, so the author concluded that the human society has shaped a small world. We understand a small world here to be a network that every pair of nodes may be reachable by a shortest path [64]. In the literature, the small world concept refers to the measure of average path length, marked $l$ above. Hence, the average path length represents the most useful measure in the complex networks analysis. Empirically, it has been discovered the value $l$ is small in large real networks like random graphs [63] [46] [40] [35]. Moreover, to show the small world effect in disconnected graphs, Latora et Marchiori has proposed to verify high value of $E_{\text {glob }}(G)$ [36].

3.1.6. Clustering coefficient. The clustering coefficient, called transitivity [12], is a property that reveals the following phenomenon seen in real networks: if node $\mathrm{A}$ is connected to node $\mathrm{B}$ and $\mathrm{B}$ to node $\mathrm{C}$ then it is more likely that $\mathrm{A}$ will also be connected to $\mathrm{C}$. In social science, this phenomenon is resumed by the famous concept 'the friend of your friend is likely also to be your friend'. In fact, the root of this idea is founded in the work of George Simmel, which introduces the concept of triad as a fundamental structure of social networks [64]. A triad represents a social group composed of three members whereas a dyad is two members. In terms of topology networks, the clustering coefficient means the high presence of triangle in a network [43]. Two definitions of clustering coefficient exist in the literature: the global clustering, and the local clustering.

Global clustering. It is defined to be three times the number of a triangle on the number of connected triples of nodes measured in the graph. Let us consider the graph $G$, the global clustering coefficient $C^{1}$ is given by expression:

$$
C^{1}=\frac{3 \times \text { number of triangles in } G}{\text { number of connected triplets in } G}
$$




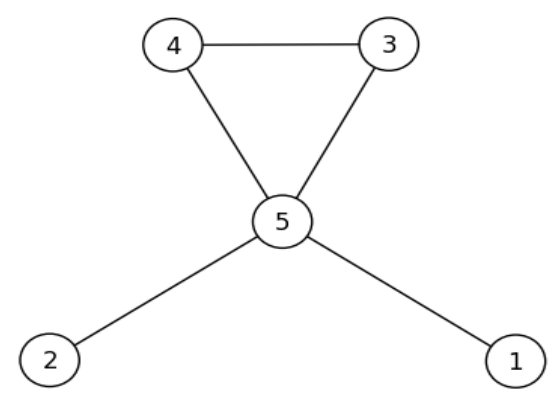

Figure 1: Illustration of clustering coefficient: $C^{1}=3 \times \frac{1}{8}=\frac{3}{8}$ because we have a single triangle and eight triplets while all the evaluated local clustering coefficient is $C_{1,2,3,4,5}=\left\{0,0,1,1, \frac{1}{6}\right\}$ which donate the mean $C^{2}=\frac{13}{30}$

The factor 3 in numerator compensates the fact that each triangle contributes to three triples and ensures that $C^{1}$ lies in the range $0 \leq C^{1} \leq 1$. If $C^{1}=1$ we have $G=K_{N}$. The formula 5 can be rewrite as the following form [43]:

$$
C^{1}=\frac{6 \times \text { number of triangles in } G}{\text { number of paths of length two }}
$$

where a path of length two refers to a directed path starting from a specified vertex.

Local clustering. It concerns a particular node $i$. The local clustering coefficient can be quantified thus:

$$
C_{i}=\frac{\text { number of connected triangles to } i}{\text { number of centred triplets on } i}
$$

Watts and Strogatz give this definition above in [63]. Let considered $e_{i}$ to be the number of edges between neighbours of $i$ and $k_{i}$ the degree of node $i$. We obtain the equation 7 otherwise.

$$
C_{i}=\frac{2 \times e_{i}}{k_{i}\left(k_{i}-1\right)}
$$

The aim of local clustering is to calculate the second clustering coefficient $C^{2}$ that is the mean of $C_{i}$

$$
C^{2}=\frac{1}{N} \sum_{i} C_{i}
$$

By definition, we have $0 \leq C_{i} \leq 1$ and $0 \leq C^{2} \leq 1$. The two definitions of clustering coefficient, $C^{1}$ and $C^{2}$, give two different values illustrated for example in figure 1 . However, the details of these differences are explained in [43]. Furthermore, the clustering coefficient have extended in weighted graphs, for example in the article of Opsahl et Pietro Panzarasa [48]. Another alternative measure of clustering property in disconnected graph is the local efficiency [36] defined by:

$$
E_{l o c}=\frac{1}{N} \sum_{i \in V} E\left(G_{i}\right)
$$

where $E\left(G_{i}\right)$ is the efficiency $G_{i}$ evaluated in formula 4 . Generally, we noticed that the presence of triples or triads in random graphs is poorer than real networks where a high clustering coefficient is observed [64].

3.1.7. Degree distribution. At first, remember that the node degree $k$ is the number of incident edges that connect this node. $P(k)$ or $p_{k}$ is defined to be the fraction of nodes of degree $k$ on the total number of nodes in the graph. The term $P(k)$ means also the probability that a node chosen randomly have 
degree $k$. Thus, we need to plot $P(k)$ of a network by drawing the corresponding histogram with node degree in $\mathrm{X}$-axis. This histogram is said degree distribution that satisfies the normalization condition:

$$
\sum_{k=0}^{\infty} P(k)=1
$$

In the case of directed graphs, we must take into account two kinds of degrees distribution such that $P\left(k^{i n}\right)$ and $P\left(k^{o u t}\right)$. The $n$-moment of degree distribution in a graph is obtained thus:

$$
\left\langle k^{n}\right\rangle=\sum_{k} k^{n} P(k)
$$

If $n$ equals one, we have the first moment $\langle k\rangle$ corresponding to the mean degree in the graph. The second moment $\left\langle k^{2}\right\rangle$ enables to measure the fluctuations of the connectivity observed in the distribution of nodes. Consequently, the divergence of $\left\langle k^{2}\right\rangle$ in the limit of infinite graph size, radically changes the behaviour of dynamic processes that take place over the graph [12].

For a long time, we had believed that the degree distribution in many networks followed Poisson distribution $\left(p_{k} \sim \frac{z^{k} e^{-z}}{k !}\right.$ where $\left.z=\langle k\rangle\right)$, in fact experiences have shown that the majority of real networks present power-law distribution $\left(p_{k} \sim k^{-\lambda}\right)$ [43]. This feature implies, in real networks, certain nodes, which have higher degree play a particular role than lower degree. We call these networks scale-free.

3.2.8. Assortativity. Indeed, the degree distribution has always been used to determine the statistical properties in non-correlated graphs. Nevertheless, it is observed that many networks present correlations between different nodes as much as the probability a node with degree $k$ connect to another node with degree $k^{\prime}$ depend on $k$ and $k^{\prime}$. It reveals necessary to introduce the notion of conditional probability $P\left(k^{\prime} \mid k\right)$, which is defined to be the probability that an edge originating from the node of degree $k$ connects the node of degree $k^{\prime} . P\left(k^{\prime} \mid k\right)$ satisfies the normalization $\sum_{k^{\prime}} P\left(k^{\prime} \mid k\right)=1$ and the balance condition [12]:

$$
k P\left(k^{\prime} \mid k\right)=k^{\prime} P\left(k \mid k^{\prime}\right)
$$

If the graph is non-correlated, $P\left(k^{\prime} \mid k\right)$ does not depend on $k$. According to the normalization and the equation 13 , we deduct the follow relation:

$$
P\left(k^{\prime} \mid k\right)=\frac{k^{\prime} P\left(k^{\prime}\right)}{\langle k\rangle}
$$

In general, the direct evaluation concerning the conditional probabilities measures give some noisy results, which are difficult to analyse in most of the real networks. To solve this problem, researchers has proposed to use the average nearest neighbours degree of vertex $i$. Let us consider the adjacent matrix of graph $G\left(a_{i j}\right)$, the average nearest neighbours degree of vertex $i, k_{n n, i}$, is defined thus:

$$
k_{n n, i}=\frac{1}{k_{i}} \sum_{j=1}^{N} a_{i j} k_{j}
$$

To better interpret the distribution of the correlation of the nodes, we define also the average degree of the nearest neighbours of a vertex of degree, $k_{n n}(k)$, called assortativity of graph. Using the equation $15, k_{n n}(k)$ is given thus:

$$
k_{n n}(k)=\frac{1}{N_{k}} \sum_{i / k_{i}=k} k_{n n, i}
$$

where $N_{k}$ is the number of nodes of degree $k$. Another definition of assortativity $k_{n n}(k)$ can be obtain by using the conditional probabilities :

$$
k_{n n}(k)=\sum_{k} k^{\prime} P\left(k^{\prime} \mid k\right)
$$


If the graph is not correlated then the above formula 17 gives $k_{n n}(k)=\left\langle k^{2}\right\rangle /\langle k\rangle$. A correlated graph is said to be 'assortative' whether $k_{n n}(k)$ increase function of $k$. In other words, the nodes highly connected tend to bind with other nodes highly connected. To illustrate it, this characteristic is observed in most social networks, it's named the popularity. Contrariwise, if $k_{n n}(k)$ decrease function of $k$ the graph is 'disassortative' that means the nodes highly connected tend to join other nodes weakly connected. We observe particularly this phenomenon in certain networks such as the Internet.

3.1.9. Centrality. The notion of centrality derives typically from the social networks area. Researchers have pointed out that certain individuals played more important roles than others in a social network. For instance, some people have more contacts inside the network compared to others [16]. Then, the measure of centrality allows to quantify the importance of a node in a graph. In fact, the centrality characterises the robustness or the vulnerability of a network. For example, destroying central nodes have many consequences on the diffusion of information in a social network. Different measures of centrality have been proposed in directed or undirected graphs. In 1979, Freeman, in his article titled Centrality in social networks: Conceptual clarification proposed three definitions of the concept of centrality: degree centrality, closeness centrality, betweenness centrality [24].

Degree centrality. The idea of this measure is that the central actors are the actors, which have more links with others. This measure considers the node degree in graph, so it is called the degree centrality. Considered the graph $G=(V, E)$ with size $N$, we can define the degree centrality of node $i, C_{D}(i)$ by:

$$
C_{D}(i)=\frac{1}{N} \sum_{j} a_{i j}
$$

where $\left(a_{i j}\right.$ is the adjacent matrix of $G$. In case of directed graphs, we should consider the in-degree and out-degree in the calculation of degree centrality. This measure of centrality is local, so it does not take into account all the structure of the graph. However, it is used in many contexts so far, like sociology [59].

Closeness centrality. It is the second measure suggested by Freeman. This measure is based on the idea, which said a node is central if it can quickly interact with all the nodes in the graph i.e. if the node is between the closeness with other nodes in the graph. In the graph $G=(V, E)$ with size $N$, the closeness centrality is calculate by:

$$
C_{C}(i)=\frac{N-1}{\sum_{j \in V \backslash\{i\}} \operatorname{dist}(i, j)}
$$

where $\operatorname{dist}(i, j)$ is the distance between $i$ and $j$. To calculate the distance $\operatorname{dist}(i, j)$, many metrics have been used in the literature. In [24], Freeman proposed to use the geodesic distance. Consequently, higher is the closeness centrality closer is the node with all other nodes. Thus, this node becomes central. In the same way, we can extend this formula in directed graph.

Betweenness centrality. The role of the betweenness centrality is to measure the usefulness of nodes to transmit information inside the network. To be more precise, a node having a high betweenness centrality is a node that passes through it numerous geodesic path (i.e. shortest path) in the graph. For instance, in information networks, the betweenness centrality is considered as the probability that the information transmitted between two nodes pass through this intermediate node [16]. We obtain its value by the following formula.

$$
C_{B}(i)=\sum_{j, k \in V, j \neq k} \frac{\sigma_{j k}(i)}{\sigma_{j k}}
$$




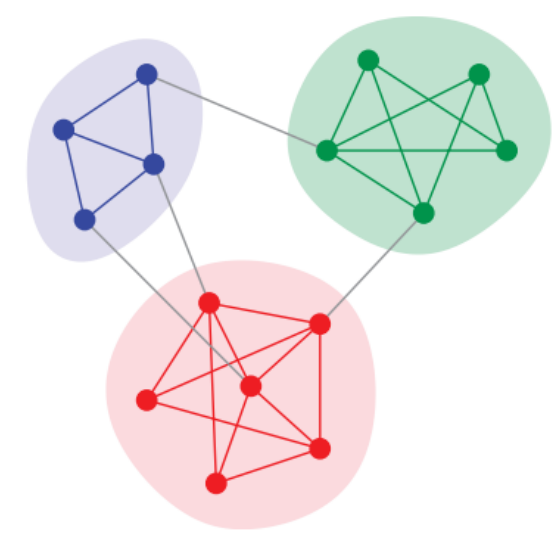

Figure 2: A small network with three community structures

where $\sigma_{j k}(i)$ is the number of the shortest path between $j$ and $k$ passing through $i$ and $\sigma_{j k}$ the total number of the shortest path between $j$ and $k$. Furthermore, an another measure of betweenness called edge-betweenness is used by Girvan and Newman to find community structure [45].

3.1.10. Community structure. Finding community structure in networks has early interested the sociologists. Intuitively, in a social network, individuals tend to regroup naturally in communities, which are constituted friendships, collaborations, hobbies, relationships, and so on. Recently, the study of community structures in networks has attracted great attention in disciplines as diverse as physics, biology, computer sciences, etc. Defining a community structure is not easy because there exist many definitions that are different according to their context [23]. However, a definition largely accepted, related to the topology of networks, defines a community structure as subgraph within which the network connections are denser than the rest of graphs (Girvan and Newman [45]). This definition indicates the densification of connectivity inside the internal nodes compared to external nodes such as showing in figure 2. In perspectives to find community structures in networks, researchers have proposed many algorithms and others are discovered every day. We remark that all the proposed techniques use generally the graph partitioning approach and some of these algorithms do not work whether the graph is disconnected [16].

Among the method of community detection discovered, we have on the one hand the methods based on traditional techniques of successive division of graphs to subset under the following criterion: the split occurs such that we preserve the more possible link for each cluster created, on the other hand those which maximize the modularity $Q^{4}$ (partition quality) introduced by Girvan and Newman [45]. As well as, Palla et al. define another approach of community structure, overlapping community, which considers that a node can belong possibly in many communities [49]. This approach come more and more to be used in the algorithms of community detection.

\subsection{Models of networks}

With the aim to create models that take into account the structure and the dynamics of complex networks, researchers have invented various synthetic models that help us to analyse their statistical properties. We focus here on the benchmark models that are Erdős and Rényi model (random graphs), Watts \& Strogatz model (small world) and Albert and Barabási model (scale free).

3.2.1. Random graphs. In 1959, Erdős and Rényi has firstly initiated the study of random graphs in

\footnotetext{
${ }^{4}$ if $e_{I J}$ is the fraction of all edges in the network that link vertices in community $I$ to vertices in community $J$ and $a_{I}=\sum_{J} e_{I J}$, which represent the fraction of edges that connect to vertices in community $I$. Then,

$$
Q=\sum_{J}\left(e_{I I}-a_{I}^{2}\right)
$$
}


their article titled On Random Graphs [21]. In this paper, the authors proposed the first random graph model with $N$ nodes and $E$ edges, it is called Erdös Rényi random graph, noted $G_{(N, E)}^{E R}$. This graph is defined from a graph without edges that is $N$ nodes entirely disconnected. Thus, a pair of nodes is selected randomly uniform to create an edge until we have $E$ links. Another alternative model, named $G_{(N, p)}^{E R}$ equivalent the precedent seems mostly studied in the community. In this latter, the process starts with $N$ nodes and every edge is chosen independently with probability $p$ or left with probability $1-p$. As a result, the graph $G_{(N, p)}^{E R}$ is entirely constructed with $p^{E}(1-p)^{N(N-1) / 2-E}$. However, It has been proved that the complexity to generate $G_{(N, p)}^{E R}$ is $O\left(N^{2}\right)$, because you may consider for each couple of vertex the presence or absence of link, while the complexity to create $G_{(N, E)}^{E R}$ is $O(E)$ [59].

Overall, the characteristics of $G_{(N, p)}^{E R}$ have often studied function of average degree $\langle k\rangle$. At the end of the procedure of generating the random graph, the expected number of edges is $\langle E\rangle=p N(N-1) / 2$ then we can deduct the average degree thus.

$$
\langle k\rangle=2\langle E\rangle / N=\frac{N(N-1) p}{N}=(N-1) p \simeq N p
$$

Erdős and Rényi were the first studying the maximum and minimum degree of a random graph while Bollobás found the complete distribution later in 1981 [4]. In random graph, the probability that a node $i$ has the degree $k=k_{i}$ follows the binomial distribution with parameters $N-1$ and $k$.

$$
P\left(k=k_{i}\right)=C_{N-1}^{k} p^{k}(1-p)^{N-1-k}
$$

where $p^{k}$ is the probability to have $k$ edges, $(1-p)^{N-1-k}$ represent the probability that the remaining $N-1-k$ attempts have not resulted in a link and $C_{N-1}^{k}$ is the number of different ways these edges may be selected. While $N \rightarrow \infty, p \rightarrow 0$, for a fixed $\langle k\rangle$ value, we have exactly the binomial distribution which tend to Poisson distribution.

$$
P\left(k=k_{i}\right) \simeq \frac{\langle k\rangle^{k}}{k !} e^{-\langle k\rangle}
$$

The reason why this homogeneous distribution is called Poisson Random Graph. Although, this distribution is exactly different from the real world networks whose distribution follows power law. The study of random networks reveals an interesting property that is the critical value phenomenon, said often the percolation. This phenomenon shows the appearance of giant components when we reach a threshold value of probability, $p_{c}=\frac{1}{N}$, corresponding to the average $\langle k\rangle_{c}=1$.

The random graphs design a typical feature founded in real networks, discussed in section 2.1.5 that is small world effect. If we consider $\operatorname{Diam}(G)$ as the diameter of a random graph $G$ we obtain its value around $\operatorname{Diam}(G) \sim \ln N / \ln (p N)=\ln N / \ln \langle k\rangle$. Consequently, the average path length $l$ is function of $N$ and has the same behaviour as the diameter, $l \sim \ln N / \ln \langle k\rangle \sim \operatorname{Diam}(G)$. Thus, the measure of $l$ appears small corresponding the small world effect. Nonetheless, other properties of random graphs are not similar with those observed in real networks. For example, the clustering coefficient varies around $C=p=\langle k\rangle / N$ and became small when $N$ is large. In addition, the degree distribution of such graphs is Poisson, which is opposite to the distributions of majority real networks. We have also no correlation between the degrees of adjacent nodes and none community structure [43].

Despite these shortcomings, Bender and Canfield suggest a generalized model, called configuration model [9]. The aim of this model is to generate a random graph with controlled degree distribution, which may be heterogeneous, i.e. we can force the degree distribution to follow power law. In 1995, Molloy and Reed define the formalization of this model [39]. In spite of heterogeneous degree, this model exhibits a small clustering coefficient and it has not any community structure.

3.2.2. Watts \& Strogatz model. It is the first model used to model the graphs, which are said small world. According to Watts and Strogatz, a small world network is characterized to have a high value 


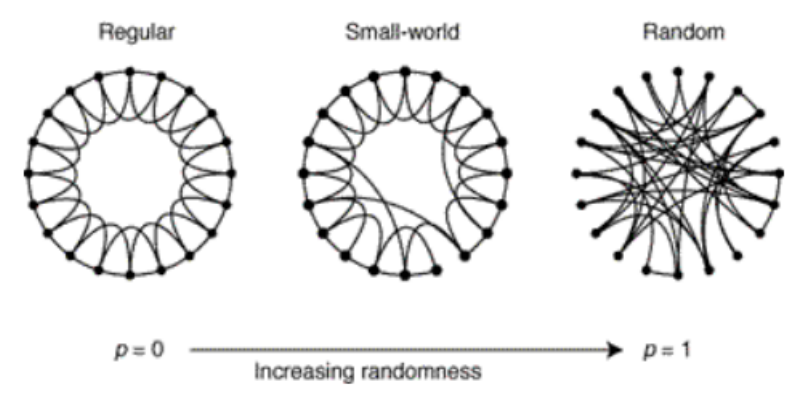

Figure 3: Rewiring procedure of Watts and Strogatz model. After [63]

of clustering coefficient $C$ and a small value of average path length $l$ [63]. The principle is, from a regular lattice to a random graph we get a graph that presents the small world characteristics. Edges are rewired according to probability $p$ (see figure 3 ). Initially, the authors start the process from a regular lattice, given $N$ fixed nodes ring, in which every node is symmetrically linked to its $2 m$ nearest neighbours. The nodes are visited one after the other, i.e. each link connecting a node to its neighbours clockwise is rewired to a randomly chosen node with probability $p$, and is left in place with probability $1-p$. We emphasize that if $p=0$ we have a regular lattice whereas in case of $p=1$, the obtained graph is completely random and gives a minimum degree equal $m$. Therefore, between these two extreme values we regard appearing a graph, which presents the small world effect and a non-trivial clustering coefficient.

To figure out the small world effect and the clustering in Watts Strogatz model (WS), we must study the behaviour of clustering coefficient $C(p)$ and the average path length $l(p)$ as function of rewiring probability $p$. In case where the ring is regular, the calculation of clustering coefficient gives a high value, $C(0)=(3 m-3) /(4 m-2)$, which tends to $3 / 4$ while $m$ becomes large. In addition, the average path length varies around $l(0) \simeq N / 4 m$, thus it implies that the regular graph has not exhibited a small world effect. If $p \rightarrow 1$, the WS model converges to random graph with mean geodesic $l(1) \simeq \ln (N) / \ln (2 m)$ and clustering coefficient $C(1) \simeq 2 m / N$. Hence, between these two steps, we remark a logarithmic drop in the value of $l(p)$ and a decreasing of $C(p)$ after a noticeable stability in a large interval of $p$. The most important thing in this experience for Watts and Strogatz is the rapid drop of the average path length $l(p)$ whereas $C(p)$ remains constant between an interval of $p$ (see figure [63]). In fact, according to the authors, the origin of the rapid drop is characterized by the appearance of shortcuts between nodes. Each shortcut created randomly implies the reachability between every farther part of the graph. Thus, it has a significant impact in the measure of average path length in the whole graph. As the original edges are randomly replaced, then the clustering coefficient keeps similarly the same value that $C(0)$ in a meaningful interval $0 \ll p \ll 1$. Consequently, the region where $1 / N \ll p \ll 1$, Watts and Strogatz observe the coexistence of the small value $l$ and a high value of the clustering coefficient $C$ that represent an important characteristic discovered mostly in real networks [4]. We can conclude that real networks are not completely random but every two nodes can be connected between them with few links.

Despite the relevant advances made in the complex networks area, the original WS model suffer some problems. Firstly, its degree distribution does not correspond to the majority reel networks distribution, but it is not a surprise, because it was not the aim of this model [43]. Secondly, the fact that the extremity of an edge is only rewired, any loop does not exist, any other edges can't add between pairs of nodes, make the calculation of the mean geodesic $l$ in the graph hardly difficult. That is the reason why many variants of Watts and Strogatz model have been proposed in the literature. Among them, we have the Newman, Moore and Watts model [46], which reproduces exactly the same model with the only difference that here we don't replace the existent edges, but new edges making shortcuts are added between two nodes with probability $p$.

3.2.3. Scale-free. The scale free networks were firstly studied by Derek de Solla Price in 1965. Price, 


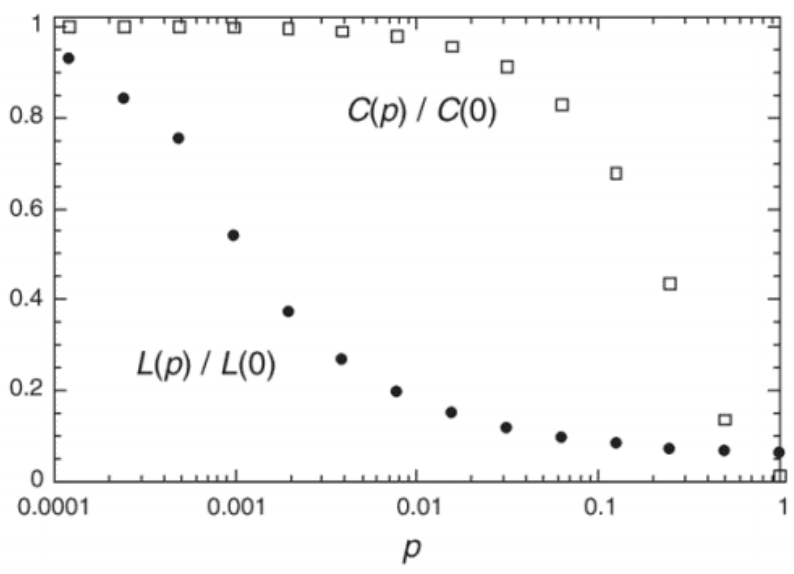

Figure 4: The characteristics of Average path length $l$ and the clustering coefficient $C$ WS model [63]

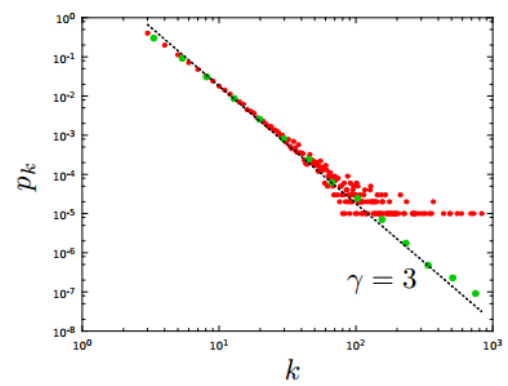

Figure 5: Generated degree distribution by Barabási-Albert model in a network with $N$ vertices and $m=3$

in his article entitled Networks of scientific papers, shows that the network of citations of scientific papers follows power law distribution [19]. Astonishingly, he found that while the network increases the degree of the nodes more connected becomes higher than the nodes less connected: he calls this phenomenon the cumulative advantage. This term is known today as preferential attachment that was introduced by Barabási and Albert [6]. The idea of Price was that the rate at which a paper gets new citations should be proportional to the number that it already has. This behaviour is observed in many real networks as the World Wide Web [43].

In 1999, Barabási and Albert created a model of graph as similar as the model discovered by Price. Contrarily to Price's model, the Barabási Albert (BA) model uses undirected edges that make to lose an important information in the network. However, if the nature of edges orientation is ignorant, the BA model allows overcoming the problem met by Price, i.e. how a paper gets its first citation (or webpage). Barabási and Albert have studied World Wide Web based on two essential rules: growth and preferential attachment [6]. These two fundamental rules are used to generate an undirected graph $G_{(N, E)}^{B A}$ from a set of vertices with initial size equal $m_{0}$. Each step $t=1,2, \ldots, N-m_{0}$ of the algorithm, a new vertex $j$ with $m \leq m_{0}$ edges is added in the network. The probability that a vertex $j$ connects with an existent vertex $i$ is linearly proportional of degree of $i$.

$$
\prod_{j \rightarrow i}=\frac{k_{i}}{\sum_{l} k_{l}}
$$

The network will have, every instant $\mathrm{t}$, a number of vertices $N=t+m_{0}$ and a number of edges $E=m t$ with a mean degree $\langle k\rangle=2 m$. Numerical simulations indicate that BA model evolve spontaneously shaping a power law distribution $P(k) \sim k^{-\lambda}$, with $\lambda=3$ (figure 5). According to the authors, the origin of power law is essentially due to the preferential attachment that leads to the appearance of 
few groups of nodes highly connected, called hubs. This small presence of hubs makes the network to be robust against random failures, but fragile to targeted attacks. It is also shown that the average path $l$ in scale free is fewer than random graphs [4].

$$
l \sim \frac{\log N}{\log \log N}
$$

The Barabási-Albert model is one of the evolving and dynamic model that displays one characteristic observed in real networks namely power law degree distribution. It is essentially the result of preferential attachment. Nevertheless, this model yields an arborescent structure that exists rarely in real networks. Thus, many modifications and extensions have been added to improve it. Among these variants, Krapivsky and Redner redefine it to directed graphs. They have also examined the existing relation between the age $a$ of a node $i$, consisting the number of nodes added after $i$, and its degree, and the correlation in degree of adjacent nodes [32]. As a result, they show the number of nodes having age $a$ with degree $k$ is:

$$
N_{a}(k)=\sqrt{1-\frac{a}{N}}\left(1-\sqrt{1-\frac{a}{N}}\right)^{k}
$$

Another alternative model is suggested by Barabási-Albert in [3]. This model is based on the following iterative algorithm: a) $m$ new nodes are added with probability $p$; b) $m$ edges are rewired with probability $q$; c) a new node is added, along with $m$ edges that connected preferentially with $m$ existent nodes according probability $1-p-q$. Similarly, the model of Dorogovtsev-Mendes-Samukhin(DMS) [20] enables to add the vertices with the following preferential attachment:

$$
\prod_{j \rightarrow i}=\frac{k_{i}+k_{0}}{\sum_{l}\left(k_{l}+k_{0}\right)} \text { with }-m<k_{0}<\infty
$$

Krapivsky et $a l$. prove that if we use the generalized preferential attachment $\prod_{j \rightarrow i}=\frac{k_{i}^{\beta}}{\sum_{l} k_{l}^{\beta}}$ the degree distribution of the network emerges in power law only if $\beta=1$. The degree distribution is exponential whether $\beta<1$ whereas a single site connects to nearly all other sites if $\beta>1$ [32].

Besides these benchmark models said above, many others models and variants have been proposed in the literature. However, because of the complexity of real networks, any of these models does not take into account all the properties of such system. Understanding at same time the topological and dynamical aspect of a complex networks remains an important challenge. Now, certain simulations give more or less pertinent outcomes in a well-defined framework that can be extended in other domains.

\section{Dynamics in complex networks}

The dynamics in networks is a crucial problematic in scientific community. In fact, most complex networks are the locus of many dynamic phenomena. We can see firstly the dynamics such that how the network evolves, i.e. appearance and disappearance of nodes and/or links, secondly the dynamics of the processes that take place in the system. The Internet and the World Wide Web have witnessed their nodes increase exponentially every day and have passed through a continuous flux of information [7]. The friendships or contact relationships in a social network can change in time because each individual may lose or have new acquaintances. In addition, the social networks have taken the place of the opinion formation, information diffusion, and rumours or epidemic spreading (more details see [50]). The telecommunication networks such as mobile phone or ad hoc networks allow collecting every day a large quantity of data. Another interesting example of evolving networks is a set of wireless mobile devices (mobile phones, laptops, sensors, ...) that are dispersed in the environment such that two devices are connected if the distance between twins is lower than a given value $R$. In this section, we try to present two modelling methods to study the dynamics in complex networks: the preferential 


\subsection{Preferential Attachment}

The Barabási-Albert model is the first model, which has used the preferential attachment to generate a time evolving network [18]. At each time step $t_{i}$, a new node is added with a number of links $m \leq m_{0}$ ( $m_{0}$ is the number of initial nodes). At the end of the process, we obtain a network with $t_{i}+m_{0}$ nodes and $m t$ links. This model described in details in section 2.2.3 have an invariant structure that is essentially determined by preferential attachment according to the authors. This is the origin of heterogeneous degrees in the network. The preferential attachment appears to be the inspiration of most dynamic graph models studied in complex networks community (see [27] [51] [18] [37]).

\subsection{Time varying graphs (TVG)}

Time varying graphs (TVG) or temporal graphs are defined as sequences of snapshots of static graphs evolving at each time step $t_{i}$. In spite of the difficulty to take into account the dynamics in a complex system, this type of graph is an adaptable mean to study the dynamic evolving in networks. We have found many acceptable descriptions of TVGs in the literature. According to Harary et al., if we add in the definition of graph $G$ described above, the function $f$ defined in a set of vertices by: $f: V \longrightarrow N$ and the function $g$ defined in a set of edges by: $g: E \longrightarrow N$, then a temporal graph $\mathcal{G}=(V, E, f, g)$ isn't nothing else the modification of one of these parameters in time [26]. Casteights et $a l$. give a clear presentation concerning the TVGs in [14]. Formally, they consider a temporal graph $\mathcal{G}=(V, E, \tau, \rho, \zeta)$ is defined such that: a set of entities $V$ (nodes), and a set of ties $E$ between entities (links), and a set of label $L$ (i.e. a label $l$ could represent link's force, social relationship intensity or type of carrier in a transportation network) such $E \subseteq V \times V \times L$, and a lifetime of the system $\tau \subseteq \mathbb{T}$ ( $\mathbb{T}$ represent time-scale which can be $\mathbb{N}$ for discrete-time systems or $\mathbb{R}$ for continuous-time systems), and a presence function $\rho: E \times \tau \longrightarrow\{0,1\}$ that indicates the given edge is available at a given time, and finally the latency function: $\zeta: E \times \tau \longrightarrow \mathbb{T}$ which stand for the delay it takes to cross a given edge if starting at a given date. On the one hand, the point of view of links the dynamic notion is given by the variation of the presence function $\rho$ and the latency function $\zeta$. On the other hand, you can see it as a successive changing of neighbours of nodes. In fact, this definition is somewhat general; it can describe a multitude of scenarios such as transport networks, information networks, social networks and so on. However, it may be contextualised about the domain that we need to study.

In the framework of a temporal network, we can add some important notions. The characteristic dates of a link $e$ corresponds to the sequence of time $t_{1}, t_{2}, t_{3}, \ldots$. The sequence $S_{\tau}(\mathcal{G})=\operatorname{sort}\left(\cup\left\{S_{\tau}(e)\right.\right.$ : $e \in E\}$ ), called set of characteristic dates of graph $\mathcal{G}$, defines the sequence of dates when topological events (appearance/disappearance of an edge) occur in the system. Each topological event can be seen as the transformation of the static graph every time step. Thus, an evolving graph $\mathcal{G}$ can be exactly defined as a sequence of static graph $G_{1}, G_{2}, G_{3}, \ldots$, where $G_{i}$ corresponds to a static snapshot of $\mathcal{G}$ at each time step $t_{i} \in S_{\tau}(\mathcal{G})$. A dynamic graph can be represented simply by one of these two structures $\left(G, S_{\mathcal{G}}, S_{\tau}\right)$ or $\left(G, S_{\mathcal{G}}, \mathbb{N}\right)$ in discrete time.

The concepts of distance, shortest path, small world effect, clustering coefficient, centrality, and community structure observed in static graphs have been extended in TVGs. For instance, in [61] the authors redefine the measures of temporal-correlation coefficient $C$, characteristic temporal path length L, and temporal global efficiency $E_{\text {glob }}$ studying the small world effect in TVGs. Moreover, Scelato et al. proposed temporal efficiency and the concept of temporal robustness as convenient measures that enable to examine the resilience in opportunist mobile networks [58]. The spreading or dissemination of information, rumour or epidemic is in an interested problem in dynamic networks. Karsai et $a l$. use the TVGs showing how the weight of a link constraint the rumour spreading within a social group [30]. Other research works try to improve the time-varying graphs modelling to better understand the dynamic phenomena observed in complex networks [66] [17] [60] [8] [52]. 


\section{Problematics and Perspectives}

The complex systems are generally the place that occur many emergent phenomena. Understanding the origin of these phenomena is a crucial problem, which has attracted the attention of researchers. In fact, the themes studying the micro and macro-meso scale have been developed in complex networks area. We have witnessed many advances, but they have mostly interested the static aspect than the dynamics aspect. Today, the availability of new sources of datasets (mobile phones records, online social networks, GPS, sensors, Bluetooth traces etc.) combined with the new modelling approaches (TVGs, variants of attachment preferential, multiplex networks) open new research opportunities in the question of dynamics in complex networks. Here we will see the research perspectives such as the mobility and community detection in dynamic networks.

- Mobility. The mobility in networks is a theme that has mostly interested the MANETS (Mobile Ad hoc Networks) area that the first models have been developed. The suggested models can be separated by two main classes: the synthetic models based on mathematical equations that try to capture the mobile motion in a simulation area [29] [42] [38] [10], and the models based on traces (logs, traceroute, geolocation etc.) obtained from systems deployed in the environment [31] [56] [28] [62]. The typical problematic in this domain is how to reconcile these two models to have a model which imitates human mobility, i.e. to create synthetic models from traces. Thus, we may ask these questions: What are the elements, which characterize human mobility? How to integrate the use of connectivity and mobility model so that we find the characteristics of human mobility [41]? As the captured traces can be heterogeneous by their sources or their formats, how to integrate them to take into account all kinds of scenarios of mobility existing in the real world? How to take into account the mobility model in dynamical processes occurring on networks such as epidemic spreading?

- Community detection in dynamic networks. The structure of the community is the notion that has been mostly studied in complex networks. Since the Newman and Girvan [45] many others algorithms of community detection continue to appear in the literature [53] [57] [34] [54] [11]. Unfortunately, the majority of them focuses on the case of static networks. Notwithstanding the efforts provided in the community detection in dynamic networks, the research activity in this domain is still an open area [25] [15] [47] [65]. Online social networks such as Facebook, Twitter, and LinkedIn witness every day communities that appear, expand, or disappear. This lead us to ask these following questions: How these communities evolve in time? What are their influences in dynamic phenomena that take place inside the network? In other words, what is the impact of community structure in the disease or rumour spreading, the diffusion or sharing of information, robustness or fragility of a network, routing protocols? How to visualize accuracy an evolving dynamic community?

\section{Conclusion}

The modelling of complex networks remains a delicate problem because of the complexity, which can occur in the structure and dynamics of these networks. However, numerous efforts have been made in the last decade. Considering that, the motivation of this research does not consist in making an exhaustive study for all the elements speaking in complex networks, but we have aimed to introduce essentially the fundamental concepts that will allow us to understand the structure and the dynamics in these networks. Additionally, we have discussed some perspectives, which can interest the scientific community. 


\section{References}

[1] L. A. Adamic and B. A. Huberman. Power-law distribution of the world wide web. Science, 2000 .

[2] R. Albert and A. L. Barab $\square$ si. Dynamics of complex systems: Scaling laws for the period of boolean networks. Phys. Rev. Lett. 84, 2000.

[3] R. Albert and A. L. Barab $\square$ si. Topology of evolving networks: Local events and universality. Phys. Rev. Lett., 2000.

[4] R. Albert and A. L. Barab $\square$ si. Statistical mechanics of complex networks. Rev. Mod. Phys., 2002.

[5] R. Albert, H. Jeong, and A. L. Barab $\square$ si. Diameter of the world-wide web. Nature 401, 1999.

[6] A. L. Barab $\square$ si and R. Albert. Emergence of scaling in random networks. Science, 1999.

[7] Alain Barrat. Les reseaux complexes et physique statistique. Archicube, 2010.

[8] P. Basu, Amotz Bar-Noy, and R. Ramanathan. Modeling and analysis of time-varying graphs. arXiv:1012.0260v1, 2010.

[9] E. A. Bender and E. R. Canfield. The asymptotic number of labelled graphs with given degree sequences. J. Comb. Theory A, 1978.

[10] C. Bettstetter. Smooth is better than sharp: a random mobility model for simulation of wireless networks. In Proceedings of MSWIM'01, 2001.

[11] V. D. Blondel, J.-L. Guillaume, R. Lambiotte, and E. Lefebvre. Fast unfolding of communities in large networks. J. Stat. Mech. P10008, 2008.

[12] S. Boccalettia, V. Latorab, Y. Morenod, M. Chavezf, and D.-U. Hwanga. Complex networks: Structure and dynamics. Physics, 2006.

[13] J. M. Buld $\square$. Applications of Complex Networks, 2012.

[14] A. Casteigts, P. Flocchini, W. Quattrociocchi, and N. Santoro. Time-varying graphs and dynamic networks. CoRR, abs/1012.0009, 2010.

[15] Y. Chen, V. Kawadia, and R. Urgaonkar. Detecting overlapping temporal community structure in time-evolving networks. arXiv:1303.7226v1 [cs.SI], 2013.

[16] N. F. CHIKHI. Calcul de centralit $\square$ et identification de structures de communaut $\square s$ dans les graphes de documents. $\mathrm{PhD}$ thesis, Universit $\square$ de Toulouse, 2010.

[17] Andrea E.F. Clementi, Claudio Macci, Angelo Monti, Francesco Pasquale, and Riccardo Silvestri. Flooding time in edge-markovian dynamic graphs. PODC'08, ACM 978-1-59593-9890/08/08, 2008.

[18] Ewan Colman. Structure and dynamics of evolving complex networks. PhD thesis, School of Information Systems, Computing and Mathematics Brunel University, 2014.

[19] Derek J. de Solla Price. Networks of scientific papers. Science, 1965.

[20] S. N. Dorogovtsev, J. F. F. Mendes, and A. N. Samukhin. Structure of growing networks with preferential linking. Phys. Rev. Lett. 85, 4633, 2000. 
[21] P. Erd $\square$ s and A. Renyi. On random graphs. Publ. Math. Debrecen, 1959.

[22] M. Faloutsos, P. Faloutsos, and C. Faloutsos. On power-law relationships of the internet topology. In ACM SIGCOMM Computer Communication Review, 1999.

[23] S. Fortunato. Community detection in graphs. Physics Reports 486, 2010.

[24] L. C. Freeman. Centrality in social networks conceptual clarification. Social Networks, page $215,1978$.

[25] Clara Granell, Richard K. Darst, Alex Arenas, Santo Fortunato, and Sergio Gómez. A benchmark model to assess community structure in evolving networks. arXiv:1501.05808v1 [physics.socph], 2015.

[26] F. Harary and G. Gupta. Dynamic graph models. Mathl. Comput. Modelling, 1997.

[27] S. A. Hill and D. Braha. Dynamic model of time-dependent complex networks. PHYSICAL REVIEW, 2010.

[28] Wei jen Hsu, T. Spyropoulos, K. Psounis, and A. Helmy. Modeling time-variant user mobility in wireless mobile networks. INFOCOM 2007. 26th IEEE International Conference on Computer Communications. IEEE, 2007.

[29] D. B. Johnson and D. A Maltz. Dynamic source routing in ad hoc wireless networks. The Kluwer International Series in Engineering and Computer Science, 1996.

[30] M. Karsai, N. Perra, and A. Vespignani. Time varying networks and the weakness of strong ties. Sci. Rep. 4, 4001; DOI:10.1038/srep04001, 2014.

[31] M. Kim, D. Kotz, and S. Kim. Extracting a mobility model from real user traces. Proceedings IEEE Infocom, 2006.

[32] P. L. Krapivsky and S. Redner. Organization of growing random networks. Phys. Rev. E 63, 2001.

[33] Ravi Kumar, Prabhakar Raghavan, Sridhar Rajagopalan, and Andrew Tomkins. Extracting largescale knowledge base from the web. Proceedings of the 9th ACM Symposium on Principles of Database Systems, 1999.

[34] A. Lancichinetti and S. Fortunato. Community detection algorithms: a comparative analysis. Physical Review E 80, 056117, 2010.

[35] M. Latapy and C. Magnien. Measuring fundamental properties of real-world complex networks. CoRR, abs/cs/0609115, 2006.

[36] V. Latora and M. Marchiori. Efficient behavior of small-world networks. PHYSICAL REVIEW LETTERS, 2001.

[37] C. Li and P. K. Maini. An evolving network model with community structure. JOURNAL OF PHYSICS A: MATHEMATICAL AND GENERAL, 2005.

[38] B. Liang and Z. J. Haas. Predictive distance-based mobility management for multidimensional pcs networks. IEEE/ACM Transactions on Networking, 2003.

[39] M. Molloy and B. Reed. A critical point for random graphs with a given degree sequence. Random Structures and Algorithms, 1995. 
[40] J. M. Montoya and R. V. Sol $\square$. Small world patterns in food webs. Journal of theoretical biology, 2000.

[41] M. Musolesi and C. Mascolo. Mobility models for systems evaluation: A survey. Springer Berlin Heidelberg, 2009.

[42] P. Nain, D. Towsley, B. Liu, and Z. Liu. Properties of random direction models. Proceedings of INFOCOM'05, 2005.

[43] M. E. J. Newman. The structure and function of complex networks. [cond-mat.stat-mech], 2003.

[44] M. E. J. Newman. Complex systems: A survey. Am. J. Phys., 2011.

[45] M. E. J. Newman and M. Girvan. Finding and evaluating community structure in networks. arXiv:cond-mat/0308217v1 [cond-mat.stat-mech], 2003.

[46] M. E. J. Newman, C. Moore, and D. J. Watts. Mean-field solution of the small-world network model. Physical Review Letters, 2000.

[47] N. P. Nguyen, T. N. Dinh, Y. Shen, and My T. Thai. Dynamic social community detection and its applications. PLoS ONE 9(4): e91431. doi:10.1371/journal.pone.0091431, 2014.

[48] T. Opsahl and P. Panzarasa. Clustering in weighted networks. Preprint submitted to Social Networks, 2009.

[49] G. Palla, I. Derenyi, I. Farkas, and Tamas Vicsek. Uncovering the overlapping community structure of complex networks in nature and society. Nature 435, 2005.

[50] R. Pastor-Satorras, C. Castellano, P. V. Mieghem, and A. Vespignani. Epidemic processes in complex networks. Rev. Mod. Phys., 2015.

[51] J. L. Payne and M. J. Eppstei. Evolutionary dynamics on scale-free interaction networks. IEEE TRANSACTIONS ON EVOLUTIONARY COMPUTATION, 2008.

[52] Nicola Perra, Andrea Baronchelli, Delia Mocanu, Bruno Gon $\square$ alves, Romualdo Pastor-Satorras, and Alessandro Vespignani. Random walks and search in time-varying networks. Physique Review Letters; 10.1103/PhysRevLett.109.238701, 2012.

[53] P. Pons and M. Latapy. Computing communities in large networks using random walks. J Graph Algor Appl 3733, 2006.

[54] U. N. Raghavan, R. Albert, and S. Kumara. Near linear time algorithm to detect community structures in large-scale networks. Phys. Rev. E 76, 2007.

[55] S. Redner. How popular is your paper? an empirical study of the citation distribution. arXiv:cond-mat/9804163v2 [cond-mat.stat-mech], 1998.

[56] Injong Rhee, Minsu Shin, Seongik Hong, Kyunghan Lee, and Song Chong. On the levy-walk nature of human mobility. INFOCOM 2008. The 27th Conference on Computer Communications. IEEE, 2008.

[57] M. Rosvall and C. T. Bergstrom. Maps of random walks on complex networks reveal community structure. Proc Natl Acad Sci 105, 2008. 
[58] S. Scellato, I. Leontiadis, C. Mascolo, P. Basu, and M. Zafer. Evaluating temporal robustness of mobile networks. Proceedings of IEEE Infocom, 2011.

[59] Massoud SEIFI. Cours stables de communaut $\square s$ dans les graphes de terrain. PhD thesis, Universit $\square$ Pierre et Marie Curie, 2012.

[60] E. Soedarmadji and R. J. McEliece. A dynamic graph algorithm for the highly dynamic network problem. PERCOMW'06, 2006.

[61] J. Tang, S. Scellato, M. Musolesi, C. Mascolo, and V. Latora. Small-world behavior in timevarying graphs. PHYSICAL REVIEW, 2010.

[62] C. Tuduce and T. Gross. A mobility model based on wlan traces and its validation. INFOCOM 2005. 24th Annual Joint Conference of the IEEE Computer and Communications Societies, 2005.

[63] Duncan J. Watts and Steven H. Strogatz. Collective dynamics of 'small-world' networks. Nature, 1998.

[64] Faraz ZAIDI. Analyse, Structure et Organisation des $R \square$ seaux Complexes. PhD thesis, UNIVERSITE BORDEAUX I, 2010.

[65] S. Zhang, J. Zhao, and X. Zhang. Co-community structure in time-varying networks. arXiv:1111.1365v2 [physics.soc-ph], 2012.

[66] S. Zhou, J. Lafferty, and L. Wasserman. Time varying undirected graphs. Mach Learn, 80:295$-319,2010$. 